\title{
Shot noise in the hybrid triple-quantum-dot interferometer coupled to superconductor
}

\section{and normal terminals}

\author{
Hong-Kang Zhao ${ }^{1,2 *}$, Jian Wang ${ }^{2}$, Qing Wang $^{1}$ \\ 1. School of Physics, Beijing Institute of Technology, Beijing 100081, China and \\ 2. Department of Physics, The University of Hong Kong, Pokfulam Road, Hong Kong, China
}

(Dated: March 20, 2014)

The shot noise of a hybrid triple-quantum-dot (TQD) interferometer has been investigated by employing the nonequilibrium Green's function method, and the general shot noise formula has been derived. The oscillation behaviors of transmission coefficients and shot noise versus the AharonovBohm phase $\phi$ exhibit asymmetric Fano resonance structure and blockade effect. Sub-Poissonian and super-Poissonian behaviors of shot noise appear in different regimes of terminal bias $e V_{\gamma}$ contributed by the Andreev reflection, and correlation of Andreev tunneling with the normal electron transport. The inverse resonance and resonance structures emerge in the shot noise and Fano factor with respect to one of the gate voltages in different regimes of $e V_{\gamma}$. The asymmetric structure can be enhanced by modifying the energy levels and gate biases of the TQD. The self-correlation and cross-correlation of current components contribute to the enhancement and suppression of shot noise.

PACS numbers: 74.50.+r,73.40.-c,73.21.-b,72.10.Bg

Keywords: hybrid triple-quantum-dot; Shot noise; Andreev reflection ; Aharonov-Bohm interferometer; current correlations

\section{Introduction}

Coupled quantum dots (QDs) induce much attention of researchers owing to the plenty physics and potential applications in nanoelectronics. Especially under the perturbation of external fields, different features may be generated from concrete construction and manipulation. Such artificial molecules provide convenient platform for revealing and demonstrating novel physics in nature. The coherent exchange and double beam splitter oscillations [1], the Coulomb blockade and Kondo-Fano resonances in single QD [2-4], double QD (DQD) [5-9] interferometers are examples in this research field. The coupled triple QD (TQD) interferometer is constructed by three QDs embedded in the loop

\footnotetext{
* Corresponding author

Electronic mail: zhaohonk@yahoo.com

Telephone : +86(10)68914975
} 
conductor under the control of an Aharonov-Bohm (AB) flux $\Phi$. The AB oscillation is induced from the accumulation of phase difference between different paths through a closed loop device. It has been demonstrated that the electronic states may be affected due to the perturbation of external AB field in the triangular TQD system [10,11]. This kind system can be used as a novel AB interferometer to obtain novel characteristics, since we have sufficient degree of freedom for controlling electrons. The destructive interference can trap an electron in a dark state that completely blocks the current through the device. Corresponding to the current correlations, the super-Poissonian noise is realized associated with the coherent transport $[12,13]$.

Shot noise is originated from higher order behavior of particle transport, and it may supply additional information beyond the current-voltage characteristics and conductance. The discreteness and randomness of particles, as well as the quantum fluctuation produce noise in the nano-devices and circuits. Generally, thermal noise, quantum noise, and shot noise are involved in the current correlation, and from the current correlation we can derive noise formulas. Shot noise describes the nonequilibrium property of discrete particles in a sample or a device at low temperature [14-16]. Since quasi-particles behave like discrete charged particles with the effective charge $q$, one can determine the charge of a quasi-particle by comparing the measurement result of current correlations with derived shot noise formula. The measurement of shot noise in quantum coherent superconductor-semiconductor junctions demonstrated the charge of Cooper pair $q=2 e[17]$, where $e$ is electron charge $e \approx 1.6 \times 10^{-19} C$. The fractional charges of quasi-particles have been observed through measuring the shot noise generated by partitioning edge currents of fractional quantum Hall systems [18-20]. The systematic measurement of shot noise in a single-electron transistor (SET) has been executed by embedding a SET in a resonance circuit [21]. The shot noise detectors have been realized through operating high-frequency signals $[22,23]$. Through investigating electron shot noise, frequency-selective single-photon detection has been contrived by employing a DQD device [24].

Andreev reflection takes place in a normal metal-superconductor (NS) hybrid junction. As an incident electron transports from normal metal to superconductor, a hole is reflected, and a Cooper pair quasi-particle with net charge $q=2 e$ is transferred through the junction [25]. The Andreev reflection opens a channel for electron to tunnel as the energy of electron is smaller than the energy gap of superconductor. Specular Andreev reflection occurs when Dirac fermions transport through a normal metal-superconductor interface in graphene, where the electron and hole occupy different valleys of the band structure [26-28]. Nonlocal Andreev reflection occurs when the separation of a tunnel barrier is comparable to the superconducting coherence length [29-31]. The entangled Andreev pairs and collective excitations in nanoscale superconductors have been investigated to provide future strategies for the 
detection of entangled electron pairs in solid-state devices [32]. Positive cross-correlation has been detected in hybrid superconductive and normal-metal three-terminal devices, from which the entangled electrons can be generated [33]. As a QD is connected to a normal metal and a superconductor, resonant transport of electron is strongly affected by the superconductor, and novel resonant tunneling properties are induced by the Andreev reflection [34,35]. The tunable Andreev reflection in an AB interferometer with four coupled QDs connecting to a normal metal and a superconductor exhibits extremely narrow resonant current peak and antiresonance current dip [36]. The Josephson current through strongly coupled DQD is adjusted by the parameters of the DQD, where the Kondo effect and the interdot superexchange coupling take important role[37]. The hybrid TQD system is investigated, where a normal metal and a superconductor are coupled to the central QD, while the other two QDs are laterally coupled to the central QD [38]. The shot noise formula has been derived by Anantram and Datta [39] through using elastic scattering matrix theory approach. The shot noise of hybrid nanodevices relating to the single QD molecular and DQD interferometer has been investigated theoretically by employing the nonequilibrium Green's function (NGF) approach [40,41].

In this Letter, we investigate the shot noise of the hybrid TQD system with three separate QDs coupled to each other, and the coupled TQD is connected to two normal metal terminals and one superconductor. Through evaluating the current correlations we have derived the general shot noise formula by employing the NGF approach. The selfcorrelation and cross-correlation of current components associated with normal electrons, quasi-particles, and Andreev reflecting particles contribute to the shot noise effectively.

\section{Model and Formalism}

Our hybrid TQD system is composed of three coupled QDs forming a triangular interferometer, where two QDs are connected to two normal terminals, and one QD is connected to a superconductive terminal. We present the system in Fig. 1 for helping to understand it graphically. The superconductor is denoted as terminal 1, and the normal metal terminals are denoted as terminal 2, and terminal 3 .

The system Hamiltonian $H$ is settled by summing up the separate sub-Hamiltonian and the tunneling term

$$
\begin{array}{r}
H=\sum_{\sigma}\left[\sum_{j=1}^{3} \varepsilon_{j} d_{j \sigma}^{\dagger} d_{j \sigma}+\sum_{i<j}\left(t_{i j} e^{i \varphi_{i j}} d_{i \sigma}^{\dagger} d_{j \sigma}+H . c .\right)\right] \\
+\sum_{\gamma k}\left[\sum_{\sigma} \varepsilon_{\gamma k} a_{\gamma, k \sigma}^{\dagger} a_{\gamma, k \sigma}-\left(\Delta_{\gamma} a_{\gamma, k \uparrow}^{\dagger} a_{\gamma,-k \downarrow}^{\dagger}\right.\right. \\
\left.\left.+\Delta_{\gamma}^{*} a_{\gamma,-k \downarrow} a_{\gamma, k \uparrow}\right)\right]+\sum_{k \sigma} \sum_{j=1}^{3}\left(T_{j k, j} a_{j, k \sigma}^{\dagger} d_{j \sigma}\right. \\
+ \text { H.c. }),
\end{array}
$$

where $d_{j \sigma}^{\dagger}$ and $d_{j \sigma}(j=1,2,3)$ are the creation and annihilation operators of electrons in the TQD, and $\varepsilon_{j}$ is the 
energy level of the $j$ th QD. The coupling constants between separate QDs satisfy $t_{i j}=t_{j i}^{*}$. The phase differences $\varphi_{i j}=-\varphi_{j i}$ are associated with the $\mathrm{AB}$ flux $\Phi$. We choose the gauge $\varphi_{12}=\varphi_{23}=\varphi_{31}=\phi / 3$, where $\phi=2 \pi \Phi / \Phi_{0}$ is the $\mathrm{AB}$ phase, and $\Phi_{0}=h / e$ is the flux quantum. In the Hamiltonian, $a_{\gamma, k \sigma}^{\dagger}, a_{\gamma, k \sigma}(\gamma=1,2,3)$ represent the creation and annihilation operators of electrons in the $\gamma$ th lead. The energy gap of the $\gamma$ th terminal is defined by $\Delta_{\gamma}=\left|\Delta_{\gamma}\right| e^{i \theta_{\gamma}}$, where $\theta_{\gamma}$ is the phase. The energy gap is zero for a normal metal $\left(\Delta_{2}=\Delta_{3}=0\right)$, and it is nonzero for the superconductive terminal $\left(\Delta_{1} \neq 0\right) . T_{j k, j}$ is the tunneling strength associated with the interaction of electrons between the $j$ th terminal and the $j$ th QD. The terminals are considered as macroscopic electron reservoirs which are described by the grand canonical ensembles when the terminals are uncoupled from the central region. In the derivations of current and noise formulas, we choose the chemical potential of the superconductor as the reference point. The bias voltage $V_{\gamma}$ between the $\gamma$ th terminal and the superconductor 1 is determined by the difference of chemical potentials $\mu_{\gamma}-\mu_{1}=e V_{\gamma}$. To proceed the derivations, we make the Bogoliubov transformation over the Hamiltonian of superconductor by introducing quasi-particles to diagonalize the superconductor.

The tunneling current in the $\gamma$ th terminal is determined by the continuity equation and Heisenberg equation [42]. Here we are interested in the tunneling currents and shot noise in the normal metal terminals. The current operator in the $\gamma$ th normal terminal is presented as $\hat{I}_{\gamma}(t)=\frac{i e}{\hbar} \sum_{k \sigma}\left[T_{\gamma k, \gamma} a_{\gamma, k \sigma}^{\dagger}(t) d_{\gamma \sigma}(t)-T_{\gamma k, \gamma}^{*} d_{\gamma \sigma}^{\dagger}(t) a_{\gamma, k \sigma}(t)\right]$, for $\gamma=2,3$, where $\hbar=h / 2 \pi$, and $h \approx 6.626 \times 10^{-34} \mathrm{~J}$.s is the Planck constant. The tunneling current in the $\gamma$ th terminal is given by taking ensemble average over the current operator above $I_{\gamma}(t)=\left\langle\hat{I}_{\gamma}(t)\right\rangle$. It is expressed by the Green's function matrices $\boldsymbol{G}_{i j \sigma}^{X}\left(t, t^{\prime}\right)$ and self-energy matrices $\boldsymbol{\Sigma}_{\gamma}^{X}\left(t_{1}, t\right)$ of the $\gamma$ th terminal in the Nambu representation

$$
\begin{aligned}
I_{\gamma}(t) & =2 e R e \sum_{\sigma} \int d t_{1}\left[\boldsymbol{G}_{\gamma \gamma \sigma}^{r}\left(t, t_{1}\right) \boldsymbol{\Sigma}_{\gamma}^{<}\left(t_{1}, t\right)\right. \\
& \left.+\boldsymbol{G}_{\gamma \gamma \sigma}^{<}\left(t, t_{1}\right) \boldsymbol{\Sigma}_{\gamma}^{a}\left(t_{1}, t\right)\right]_{11} .
\end{aligned}
$$

The Green's function matrices $\boldsymbol{G}_{i j \sigma}^{X}\left(t, t^{\prime}\right)$ of coupled TQD in the Nambu representation are defined by

$$
\boldsymbol{G}_{i j \sigma}^{X}\left(t, t^{\prime}\right)=\left(\begin{array}{cc}
\left\langle\left\langle d_{i \sigma}(t), d_{j \sigma}^{\dagger}\left(t^{\prime}\right)\right\rangle\right\rangle^{X}, & \left\langle\left\langle d_{i \sigma}(t), d_{j \bar{\sigma}}\left(t^{\prime}\right)\right\rangle\right\rangle^{X} \\
\left\langle\left\langle d_{i \bar{\sigma}}^{\dagger}(t), d_{j \sigma}^{\dagger}\left(t^{\prime}\right)\right\rangle\right\rangle^{X}, & \left\langle\left\langle d_{i \bar{\sigma}}^{\dagger}(t), d_{j \bar{\sigma}}\left(t^{\prime}\right)\right\rangle\right\rangle^{X}
\end{array}\right),
$$

where $X \in\{r, a,<,>\}$ represents the retarded, advanced and Keldysh Green's function matrices. The steady state tunneling current is described by the Fourier transformation of the Green's function matrices and self-energy matrices of the $\gamma$ th terminal. The subscripts $i, j$ denote the three separate QDs to form matrix blocks in the Nambu representation. The self-energy matrices of the terminals $\boldsymbol{\Sigma}_{i j}^{X}(\epsilon)$ are directly related to the connected terminal of corresponding QD, and only the diagonal elements of terminal self-energy matrices exist in our system, i.e., $\boldsymbol{\Sigma}_{i j}^{X}(\epsilon)=\boldsymbol{\Sigma}_{i}^{X}(\epsilon) \delta_{i j}$. In 
the wide-band limit, the linewidth of the $\gamma$ th normal terminal is a constant $\Gamma_{\gamma}$. Generally, the retarded self-energy matrix of the $\gamma$ th terminal is given by $\boldsymbol{\Sigma}_{\gamma}^{r}(\epsilon)=-\frac{i}{2} \Gamma_{\gamma} \boldsymbol{\rho}_{\gamma}(\epsilon)$, where the matrix $\boldsymbol{\rho}_{\gamma}(\epsilon)$ is defined as

$$
\boldsymbol{\rho}_{\gamma}(\epsilon)=\left(\begin{array}{cc}
N_{\gamma}\left(\epsilon_{\gamma}^{+}\right), & w_{\gamma}\left(\epsilon_{\gamma}^{+}\right) \\
w_{\gamma}^{*}\left(\epsilon_{\gamma}^{-}\right), & N_{\gamma}\left(\epsilon_{\gamma}^{-}\right)
\end{array}\right),
$$

with $\epsilon_{\gamma}^{\mp}=\epsilon \mp \mu_{\gamma 1}$. Here $\mu_{\gamma 1}$ is the chemical potential difference between terminals $\gamma$ and 1 stated as $\mu_{\gamma 1}=\mu_{\gamma}-\mu_{1}$, and the voltage between the two corresponding terminals $V_{\gamma}$ is settled by $\mu_{\gamma 1}=e V_{\gamma}$. The functions $N_{\gamma}(\epsilon)$ and $w_{\gamma}(\epsilon)$ are defined by $N_{\gamma}(\epsilon)=|\epsilon| / \sqrt{\epsilon^{2}-\left|\Delta_{\gamma}\right|^{2}}, \quad w_{\gamma}(\epsilon)=\Delta_{\gamma} / \sqrt{\epsilon^{2}-\left|\Delta_{\gamma}\right|^{2}}$, which contribute effectively to the mesoscopic transport in the regimes $|\epsilon|>\left|\Delta_{\gamma}\right|$, and $|\epsilon|<\left|\Delta_{\gamma}\right|$. The Keldysh self-energy of the $\gamma$ th terminal is determined by $\boldsymbol{\Sigma}_{\gamma}^{<,>}(\epsilon)= \pm i \Gamma_{\gamma} \boldsymbol{\rho}_{\gamma}^{<,>}(\epsilon)$, where the matrix $\boldsymbol{\rho}_{\gamma}^{<,>}(\epsilon)$ is defined as

$$
\boldsymbol{\rho}_{\gamma}^{<,>}(\epsilon)=\left(\begin{array}{ll}
\tilde{N}_{\gamma}\left(\epsilon_{\gamma}^{+}\right) f^{<,>}\left(\epsilon_{\gamma}^{+}\right), & \widetilde{w}_{\gamma}\left(\epsilon_{\gamma}^{+}\right) f^{<,>}\left(\epsilon_{\gamma}^{+}\right) \\
\widetilde{w}_{\gamma}^{*}\left(\epsilon_{\gamma}^{-}\right) f^{<,>}\left(\epsilon_{\gamma}^{-}\right), & \widetilde{N}_{\gamma}\left(\epsilon_{\gamma}^{-}\right) f^{<,>}\left(\epsilon_{\gamma}^{-}\right)
\end{array}\right),
$$

with $\epsilon_{\gamma}^{\mp}=\epsilon \mp \mu_{\gamma 1}$, and $f^{<}(\epsilon)=f(\epsilon), f^{>}(\epsilon)=1-f(\epsilon)$. The Fermi distribution function of quasi-particles $f(\epsilon)=$ $1 /\left[\exp \left(\epsilon / K_{B} T\right)+1\right]$ is included in the self-energy matrix of terminal . The functions $\widetilde{N}_{\gamma}(\epsilon)$ and $\widetilde{w}_{\gamma}(\epsilon)$ are associated with the density of state (DOS) of the superconductor defined by $\widetilde{N}_{\gamma}(\epsilon)=\theta\left(|\epsilon|-\left|\Delta_{\gamma}\right|\right)|\epsilon| / \sqrt{\epsilon^{2}-\left|\Delta_{\gamma}\right|^{2}}$, $\widetilde{w}_{\gamma}(\epsilon)=$ $\theta\left(|\epsilon|-\left|\Delta_{\gamma}\right|\right) \Delta_{\gamma} / \sqrt{\epsilon^{2}-\left|\Delta_{\gamma}\right|^{2}}$. For the normal terminals, we have $N_{\gamma}(\epsilon)=\widetilde{N}_{\gamma}(\epsilon)=1$, and $w_{\gamma}(\epsilon)=\widetilde{w}_{\gamma}(\epsilon)=0$. The Keldysh Green's function matrices of the system $\boldsymbol{G}_{\gamma \gamma^{\prime} \sigma}^{<,>}(\epsilon)$ are determined by the retarded and advanced Green's function matrices $\boldsymbol{G}_{\gamma \ell \sigma}^{r(a)}(\epsilon)$ through the relation $\boldsymbol{G}_{\gamma \gamma^{\prime} \sigma}^{<,>}(\epsilon)=\sum_{\ell} \boldsymbol{G}_{\gamma \ell \sigma}^{r}(\epsilon) \boldsymbol{\Sigma}_{\ell \ell}^{<,>}(\epsilon) \boldsymbol{G}_{\ell \gamma^{\prime} \sigma}^{a}(\epsilon)$, where the summation with respect to $\ell$ runs over the three terminals. The Keldysh, retarded and advanced Green's function matrices abide by the relation $\boldsymbol{G}_{\gamma \gamma^{\prime} \sigma}^{r}(\epsilon)-\boldsymbol{G}_{\gamma \gamma^{\prime} \sigma}^{a}(\epsilon)=\boldsymbol{G}_{\gamma \gamma^{\prime} \sigma}^{>}(\epsilon)-\boldsymbol{G}_{\gamma \gamma^{\prime} \sigma}^{<}(\epsilon)=-i\left[\boldsymbol{G}_{\sigma}^{r}(\epsilon) \boldsymbol{\Gamma}(\epsilon) \boldsymbol{G}_{\sigma}^{a}(\epsilon)\right]_{\gamma \gamma^{\prime}}$, where $\boldsymbol{\Gamma}(\epsilon)=\sum_{\gamma} \boldsymbol{\Gamma}_{\gamma}(\epsilon)$, and $\boldsymbol{\Gamma}_{\gamma}(\epsilon)=\Gamma_{\gamma} \boldsymbol{\rho}_{\gamma}(\epsilon)$ is the $2 \times 2$ matrix in the Nambu representation. If we obtain the retarded Green's function matrix, the advanced Green's function matrix is settled by the retarded Green's function matrix through $\boldsymbol{G}_{\sigma}^{a}(\epsilon)=\boldsymbol{G}_{\sigma}^{r}(\epsilon)^{\dagger}$.

To proceed the investigation of transport behaviors for our hybrid system, we employ the equation of motion (EOM) method to derive the Green's function matrices. We define the interaction matrix $\mathcal{T}_{i j}$ and the Green's function matrix $\boldsymbol{g}_{d_{i}}^{r}(\epsilon)$ of each isolated QD in the Nambu representation as

$$
\mathcal{T}_{i j}=\left(\begin{array}{cc}
t_{i j} e^{i \varphi_{i j}} & 0 \\
0 & -t_{i j}^{*} e^{-i \varphi_{i j}}
\end{array}\right), \boldsymbol{g}_{d_{i}}^{r}(\epsilon)=\left(\begin{array}{cc}
\frac{1}{\epsilon-\varepsilon_{i}+i \eta} & 0 \\
0 & \frac{1}{\epsilon+\varepsilon_{i}+i \eta}
\end{array}\right)
$$

In the Nambu representation, the EOM of the retarded Green's function matrix is expressed by

$$
\begin{aligned}
\boldsymbol{G}_{i j \sigma}^{r}(\epsilon)= & \boldsymbol{g}_{d_{i}}^{r}(\epsilon) \delta_{i j}+\boldsymbol{g}_{d_{i}}^{r}(\epsilon)\left[\mathcal{T}_{i i-1} \boldsymbol{G}_{i-1 j \sigma}^{r}(\epsilon)\right. \\
& \left.+\boldsymbol{\mathcal { T }}_{i i+1} \boldsymbol{G}_{i+1 j \sigma}^{r}(\epsilon)+\boldsymbol{\Sigma}_{i}^{r}(\epsilon) \boldsymbol{G}_{i j \sigma}^{r}(\epsilon)\right]
\end{aligned}
$$


where $i, j=1,2,3$. Due to the coupling of the QDs, the elements of Green's function matrix are coupled to each other. The normal and abnormal Green's functions are involved in the recurrence equation. Writing out the concrete equations for separate elements of Green's function matrix, and solving the equations consistently, we can obtain the retarded Green's function matrix.

From Eq. (2), one can derive the steady state tunneling current formula in the $\gamma$ th normal terminal

$$
\begin{aligned}
I_{\gamma}=\frac{e}{h} \sum_{\beta \sigma} \int & d \epsilon\left\{T_{\gamma \beta}(\epsilon)\left[f\left(\epsilon_{\gamma}^{+}\right)-f\left(\epsilon_{\beta}^{+}\right)\right]\right. \\
& \left.+T_{\gamma \beta}^{A}(\epsilon)\left[f\left(\epsilon_{\beta}^{+}\right)-f\left(\epsilon_{\beta}^{-}\right)\right]\right\} .
\end{aligned}
$$

The transmission coefficients $T_{\gamma \beta}(\epsilon)$ represent the transport of electrons and quasi-particles from the $\gamma$ th terminal to the $\beta$ th terminal $(\beta \neq \gamma)$ defined by $T_{\gamma \beta}(\epsilon)=\left[\boldsymbol{\Gamma}_{\gamma}(\epsilon) \boldsymbol{G}_{\gamma \beta \sigma}^{r}(\epsilon) \boldsymbol{\Gamma}_{\beta}(\epsilon) \boldsymbol{G}_{\beta \gamma \sigma}^{a}(\epsilon)\right]_{11}$. The transmission coefficients consist of electron tunneling from the $\gamma$ th normal terminal to the other normal terminal, and the transport of quasi-particles to the superconductive terminal. The quasi-particles transporting through the superconductor require that their energies are restricted by $|\epsilon|>\Delta$, where $\Delta=\left|\Delta_{1}\right|$. The transmission coefficients $T_{\gamma \beta}^{A}(\epsilon)$ describe the Andreev reflection of electron from the $\gamma$ th normal terminal back to the same normal terminal for $\beta=\gamma$, and to the other normal terminal for $\beta \neq \gamma$ defined by $T_{\gamma \beta}^{A}(\epsilon)=\boldsymbol{\Gamma}_{\gamma, 11}(\epsilon) \boldsymbol{G}_{\gamma \beta \sigma, 12}^{r}(\epsilon) \boldsymbol{\Gamma}_{\beta, 22}(\epsilon) \boldsymbol{G}_{\beta \gamma \sigma, 21}^{a}(\epsilon)$, where $\beta \neq 1$. The electrons with energy $|\epsilon|<\Delta$ and $|\epsilon| \geq \Delta$ can contribute to the Andreev tunneling current.

Shot noise $S_{\gamma \gamma^{\prime}}(\Omega)$ is generated by the current correlation defined by the relation $2 \pi S_{\gamma \gamma^{\prime}}(\Omega) \delta\left(\Omega+\Omega^{\prime}\right)=$ $\left\langle\delta \hat{I}_{\gamma}(\Omega) \delta \hat{I}_{\gamma^{\prime}}\left(\Omega^{\prime}\right)\right\rangle+\left\langle\delta \hat{I}_{\gamma^{\prime}}\left(\Omega^{\prime}\right) \delta \hat{I}_{\gamma}(\Omega)\right\rangle$, where $\delta \hat{I}_{\gamma}(\Omega)=\hat{I}_{\gamma}(\Omega)-\left\langle\hat{I}_{\gamma}(\Omega)\right\rangle$ is the current fluctuation operator [14]. $\Omega, \Omega^{\prime}$ represent the frequencies related to the Fourier transformation over times $t$ and $t^{\prime}$. Substituting the current operator into the current correlation formula, and employing the Wick's theorem we can express the current correlations by Green's function matrices. The normal and abnormal operator correlations contribute to shot noise due to the coupling of superconductor. Through some technical evaluations we express shot noise by the Green's function matrices of the central coupled QDs. The shot noise related to the normal terminals $\gamma$ and $\gamma^{\prime}$ is determined by 
$S_{\gamma \gamma^{\prime}}(\Omega)=2 S_{\gamma \gamma^{\prime}}^{(1)}(\Omega)-S_{\gamma \gamma^{\prime}}^{(+)}(\Omega)$, where we have defined the functions $S_{\gamma \gamma^{\prime}}^{(1)}(\Omega)$ and $S_{\gamma \gamma^{\prime}}^{(+)}(\Omega)$ as the following forms

$$
\begin{aligned}
S_{\gamma \gamma^{\prime}}^{(1)}(\Omega) & =-\frac{2 e^{2}}{h} R e \int d \epsilon \sum_{\sigma}\left\{\boldsymbol{W}_{\gamma \gamma^{\prime}, 11}^{>}(\tilde{\epsilon}) \boldsymbol{W}_{\gamma^{\prime} \gamma, 11}^{<}(\epsilon)\right. \\
& +\widetilde{\boldsymbol{W}}_{\gamma \gamma^{\prime}, 11}^{>}(\tilde{\epsilon}) \widetilde{\boldsymbol{W}}_{\gamma^{\prime} \gamma, 11}^{<}(\epsilon)-\boldsymbol{G}_{\gamma \gamma^{\prime} \sigma, 11}^{>}(\tilde{\epsilon}) \boldsymbol{Q}_{\gamma^{\prime} \gamma, 11}^{<}(\epsilon) \\
& -\boldsymbol{Q}_{\gamma \gamma^{\prime}, 11}^{>}(\tilde{\epsilon}) \boldsymbol{G}_{\gamma^{\prime} \gamma \sigma, 11}^{<}(\epsilon)-\delta_{\gamma \gamma^{\prime}}\left[\boldsymbol{G}_{\gamma \gamma \sigma}^{>}(\tilde{\epsilon}) \boldsymbol{\Sigma}_{\gamma}^{<}(\epsilon)\right. \\
& \left.\left.+\boldsymbol{\Sigma}_{\gamma}^{>}(\tilde{\epsilon}) \boldsymbol{G}_{\gamma \gamma \sigma}^{<}(\epsilon)\right]_{11}\right\}, \\
S_{\gamma \gamma^{\prime}}^{(+)}(\Omega) & =-\frac{2 e^{2}}{h} \operatorname{Re} \int d \epsilon \sum_{\sigma}\left\{\boldsymbol{W}_{\gamma \gamma^{\prime}}^{>}(\tilde{\epsilon}) \boldsymbol{W}_{\gamma^{\prime} \gamma}^{<}(\epsilon)\right. \\
& +\widetilde{\boldsymbol{W}}_{\gamma \gamma^{\prime}}^{>}(\tilde{\epsilon}) \widetilde{\boldsymbol{W}}_{\gamma^{\prime} \gamma}^{<}(\epsilon)-\boldsymbol{G}_{\gamma \gamma^{\prime} \sigma}^{>}(\tilde{\epsilon}) \boldsymbol{Q}_{\gamma^{\prime} \gamma}^{<}(\epsilon) \\
& -\boldsymbol{Q}_{\gamma \gamma^{\prime}}^{>}(\tilde{\epsilon}) \boldsymbol{G}_{\gamma^{\prime} \gamma \sigma}^{<}(\epsilon)-\delta_{\gamma \gamma^{\prime}}\left[\boldsymbol{G}_{\gamma \gamma \sigma}^{>}(\tilde{\epsilon}) \boldsymbol{\Sigma}_{\gamma}^{<}(\epsilon)\right. \\
& \left.\left.+\boldsymbol{\Sigma}_{\gamma}^{>}(\tilde{\epsilon}) \boldsymbol{G}_{\gamma \gamma \sigma}^{<}(\epsilon)\right]\right\}_{11}^{<},
\end{aligned}
$$

with $\tilde{\epsilon}=\epsilon+\hbar \Omega$. In above formulas we have defined the matrices by $\boldsymbol{Q}_{\gamma^{\prime} \gamma}^{<,>}(\epsilon)=\boldsymbol{\Sigma}_{\gamma^{\prime}}^{r}(\epsilon) \boldsymbol{G}_{\gamma^{\prime} \gamma \sigma}^{r}(\epsilon) \boldsymbol{\Sigma}_{\gamma}^{<,>}(\epsilon)+$ $\widetilde{\boldsymbol{W}}_{\gamma^{\prime} \gamma}^{<,>}(\epsilon) \boldsymbol{\Sigma}_{\gamma}^{a}(\epsilon), \boldsymbol{W}_{\gamma^{\prime} \gamma}^{<,>}(\epsilon)=\boldsymbol{G}_{\gamma^{\prime} \gamma \sigma}^{r}(\epsilon) \boldsymbol{\Sigma}_{\gamma}^{<,>}(\epsilon)+\boldsymbol{G}_{\gamma^{\prime} \gamma \sigma}^{<,>}(\epsilon) \boldsymbol{\Sigma}_{\gamma}^{a}(\epsilon)$, and $\widetilde{\boldsymbol{W}}_{\gamma^{\prime} \gamma}^{<,>}(\epsilon)=\boldsymbol{\Sigma}_{\gamma^{\prime}}^{r}(\epsilon) \boldsymbol{G}_{\gamma^{\prime} \gamma \sigma}^{<,>}(\epsilon)+\boldsymbol{\Sigma}_{\gamma^{\prime}}^{<,>}(\epsilon) \boldsymbol{G}_{\gamma^{\prime} \gamma \sigma}^{a}(\epsilon)$ for writing the formulas compactly. In general, Eqs. (5) and (6) contain the information of cross-correlation noise $\left(\gamma \neq \gamma^{\prime}\right)$, and self-correlation noise $\left(\gamma=\gamma^{\prime}\right)$ at arbitrary frequency $\Omega$. Through substituting the obtained Green's function matrices into above formulas one can completely determine the shot noise. In this Letter, we only present the derivation of self-correlation noise at zero frequency $\Omega=0$, i.e., $S_{\gamma \gamma}=S_{\gamma \gamma}(0)$. Thermal noise is also included in the above noise formula, and it vanishes as temperature approaches zero. As a result of derivation, we obtain the shot noise at zero temperature

$$
\begin{array}{r}
S_{\gamma \gamma}=\frac{2 e^{2}}{h} \int d \epsilon \sum_{\sigma}\left\{\sum_{\beta \neq \gamma} T_{\gamma \beta}(\epsilon)\left[1-\sum_{\beta^{\prime} \neq \gamma} T_{\gamma \beta^{\prime}}(\epsilon)\right]\right. \\
\times F\left(\epsilon_{\gamma}^{+}, \epsilon_{\beta}^{+}\right)+2 T_{\gamma \gamma^{\prime}}^{A}(\epsilon) T_{\gamma \gamma^{\prime}}^{Q}(\epsilon) F\left(\epsilon_{\gamma^{\prime}}^{-}, \epsilon_{\gamma^{\prime}}^{+}\right) \\
+2 T_{\gamma \gamma}^{A}(\epsilon)\left[1-T_{\gamma \gamma}^{A}(\epsilon)-\sum_{\beta \neq \gamma} T_{\gamma \beta}(\epsilon)\right] F\left(\epsilon_{\gamma}^{-}, \epsilon_{\gamma}^{+}\right) \\
\left.+T_{\gamma 1}(\epsilon) T_{\gamma \gamma^{\prime}}(\epsilon) F\left(\epsilon, \epsilon_{\gamma^{\prime}}^{+}\right)\right\},
\end{array}
$$

where $\gamma$ and $\gamma^{\prime}\left(\gamma \neq \gamma^{\prime}\right)$ denote different normal terminals. We have defined the function $F\left(\epsilon, \epsilon^{\prime}\right)=f>(\epsilon) f^{<}\left(\epsilon^{\prime}\right)+$ $f^{<}(\epsilon) f^{>}\left(\epsilon^{\prime}\right)$, and the transmission coefficient $T_{\gamma \gamma^{\prime}}^{Q}(\epsilon)=\Gamma_{\gamma} \Gamma_{\gamma^{\prime}}\left|\boldsymbol{G}_{\gamma \gamma^{\prime} \sigma, 11}^{\mathrm{r}}(\epsilon)\right|^{2}$. The term contains $F\left(\epsilon_{\gamma}^{+}, \epsilon_{\beta}^{+}\right)$describes the contribution of electrons and quasi-particles transporting from the $\gamma$ th normal terminal to the other normal and superconductive terminals. This behavior induces the suppression of shot noise. The term accompanying $F\left(\epsilon_{\gamma^{\prime}}^{-}, \epsilon_{\gamma^{\prime}}^{+}\right)$ describes the noise contributed by the Andreev reflection and transport of electrons from the $\gamma$ th normal terminal to 
the $\gamma^{\prime}$ th normal terminal. This effect is ascribed to the correlation of Andreev reflecting current and normal tunneling current between the two normal terminals, which enhances the shot noise. The term containing $F\left(\epsilon_{\gamma}^{-}, \epsilon_{\gamma}^{+}\right)$is the contribution of Andreev reflecting current in the $\gamma$ th normal terminal. The correlations of Andreev reflecting current with the transporting currents coming from different terminals suppress shot noise. The last term associated with $F\left(\epsilon, \epsilon_{\gamma^{\prime}}^{+}\right)$describes the correlation of electron and quasi-particle transport, which results in the enhancement of shot noise.

A special case of the shot noise formula Eq. (7) is given by restricting the transport behavior in the regime $e V_{\gamma}=e V_{\gamma^{\prime}}=e V$, and $|e V|<\Delta$. The normal tunneling current correlation disappears, and the current correlations of Andreev reflection and electron transport in different normal terminals $\gamma$ and $\gamma^{\prime}$ determine shot noise

$$
\begin{aligned}
S_{\gamma \gamma}=\frac{4 e^{2}}{h} & \int d \epsilon \sum_{\sigma}\left\{T_{\gamma \gamma}^{A}(\epsilon)\left[1-T_{\gamma \gamma}^{A}(\epsilon)-\sum_{\beta \neq \gamma} T_{\gamma \beta}(\epsilon)\right]\right. \\
& \left.\times F\left(\epsilon_{\gamma}^{-}, \epsilon_{\gamma}^{+}\right)+T_{\gamma \gamma^{\prime}}^{A}(\epsilon) T_{\gamma \gamma^{\prime}}^{Q}(\epsilon) F\left(\epsilon_{\gamma^{\prime}}^{-}, \epsilon_{\gamma^{\prime}}^{+}\right)\right\}
\end{aligned}
$$

where $\gamma \neq \gamma^{\prime}$, and $\gamma, \gamma^{\prime}=2,3$. In Eq. (8), the quasi-particle transport associated with $T_{\gamma 1}(\epsilon)$ disappears, since the applied source-drain bias $|e V|>\Delta$ is required to overcome the threshold of superconductor for the quasi-particles. The transmission coefficient $T_{\gamma \gamma^{\prime}}(\epsilon)$ contains the normal electron transmission and Andreev reflection of electrons between the two normal terminals $\gamma$ and $\gamma^{\prime}$ determined by $T_{\gamma \gamma^{\prime}}^{Q}(\epsilon)$ and $T_{\gamma \gamma^{\prime}}^{A}(\epsilon)$, respectively. Compared with the two terminal hybrid system, here the transmission of electrons between different normal terminals enlarges the shot noise of our hybrid three terminal TQD interferometer.

As the voltages of two normal terminals are equal $e V_{2}=e V_{3}=e V$, the two normal metal terminals together take the role of source, and the superconductive terminal is the drain of TQD. At zero temperature and $|e V|<\Delta$, the tunneling current is completely induced by the Andreev reflection

$$
I_{\gamma}=\frac{e}{h} \sum_{\beta \sigma} \int d \epsilon T_{\gamma \beta}^{A}(\epsilon)\left[f\left(\epsilon_{\beta}^{+}\right)-f\left(\epsilon_{\beta}^{-}\right)\right],
$$

where $\beta=2,3$. The tunneling current is contributed by the Andreev reflection described by the transmission of electrons in the same normal terminal $T_{\gamma \gamma}^{A}$, and the transmission between different normal terminals $T_{\gamma \gamma^{\prime}}^{A}$. Shot noise in this regime is contributed by: (a) self-correlation of Andreev currents in the same terminal; (b) cross-correlations among Andreev reflecting currents in different normal terminals; (c) cross-correlation of Andreev current and normal electron current in different normal terminals.

Another special case of our system is the TQD coupled to one normal and one superconductive terminals. The transmission coefficients of the quasi-particles disappear when the source-drain bias $|\mathrm{eV}|<\Delta$ at zero temperature, 
and the shot noise formula reduces to

$$
S_{\gamma \gamma}=\frac{4 e^{2}}{h} \int d \epsilon \sum_{\sigma} T_{\gamma \gamma}^{A}(\epsilon)\left[1-T_{\gamma \gamma}^{A}(\epsilon)\right] F\left(\epsilon_{\gamma}^{-}, \epsilon_{\gamma}^{+}\right) .
$$

The Fano factor is defined by $F_{\gamma}=S_{\gamma \gamma} / 2 q I_{\gamma}$, where $q=2 e$ is the charge of Cooper pair in the superconductor. The shot noise in this regime is completely generated by the Andreev reflecting current, and it is related to the sub-Poissonian shot noise with $F_{\gamma}<1$. However, for the hybrid three terminal TQD system, the shot noise described by Eq. (7) is enhanced sufficiently to exhibit super-Poissonian shot noise with $F_{\gamma}>1$.

\section{Numerical calculations}

In this section we perform the numerical calculations on the transmission coefficients, shot noise, and Fano factor of terminal $2(\gamma=2)$ at zero temperature. We choose the energy gap of superconductor as energy scale by setting $\Delta=1$, and the shot noise is scaled by $S_{0}=4 e^{2} / h$. We consider the symmetric coupling constants as $t_{12}=t_{13}=t_{23}=0.8$, $\Gamma_{1}=\Gamma_{2}=\Gamma_{3}=0.3$. The gate voltages $V_{g j}$ are introduced in the three separate QDs by replacing the energy levels of QDs as $\varepsilon_{j} \rightarrow E_{j}-e V_{g j}$, where $E_{j}$ is the energy of the $j$ th QD. We investigate the modification of transport characteristics by adjusting the energy levels, gate voltages, AB phase, and terminal bias voltages.

In Fig. 2, we present the transmission coefficient $T_{22}^{A}$ at the incident energy of electrons $\epsilon=0.9$. The Fano peakvalley resonant structures appear periodically with respect to the AB phase $\phi$. In general, the period of oscillation is $2 \pi$ shown by the dotted and circled curves. However, for the completely symmetric situation as $E_{1}=E_{2}=E_{3}=0.1$, and $e V_{g 1}=e V_{g 2}=e V_{g 3}=0.1$, the transmission coefficient $T_{22}^{A}$ oscillates with period $\pi$ shown by the solid curve. The oscillation period of Andreev reflection in the same normal terminal may be controlled from $2 \pi$ to $\pi$ by adjusting the parameters from asymmetric to completely symmetric system. As the energy levels and gate voltages of QDs take several different values, one of the peaks in a period is suppressed, and the valleys are raised to display one higher and one lower Fano-type oscillation with period $2 \pi$. The transmission $T_{22}^{A}$ may be forbidden at $\phi=\pi(2 n+1)$, $(n=0, \pm 1, \pm 2, \ldots)$ shown by the dotted curve as we adjust the parameters.

The transmission coefficients associated with the Andreev reflection in the same normal terminal $T_{\gamma \gamma}^{A}(\phi)$, and between different normal terminals $T_{\gamma \gamma^{\prime}}^{A}(\phi)\left(\gamma \neq \gamma^{\prime}\right)$ behave quite differently. Fig. 3 shows the transmission coefficient $T_{23}^{A}$, which represents the Andreev reflection of electrons from terminal 2 to terminal 3. The specific energy level of each QD affects the transport behaviors of electrons. Through adjusting the applied gate voltages $V_{g j}(j=1,2,3)$ concrete characteristics are exhibited. For the completely symmetric situation with equal energy levels and equal gate voltages, the oscillation structure appears two lager peaks and two smaller peaks in a period $2 \pi$ (the solid curve). The enhancement of asymmetric structure $\left(E_{1}=0.1, E_{2}=-0.1, E_{3}=0.2\right.$, and $\left.e V_{g 1}=e V_{g 2}=e V_{g 3}=0.1\right)$ results in 
the suppression of the dips to zero at $\phi=\pi(2 n+1),(n=0, \pm 1, \pm 2, \ldots)$, and the Fano peak-valley resonant oscillation becomes more obvious (the dotted curve). For the completely asymmetric situation $\left(E_{1}=0.1, E_{2}=-0.1, E_{3}=0.2\right.$, $\left.e V_{g 1}=0.3, e V_{g 2}=0.1, e V_{g 3}=-0.3\right)$ shown by the circled curve, the magnitude of transmission coefficient $T_{23}^{A}$ reduces, and the zeros exhibit periodically at around $\phi=2 n \pi,(n=0, \pm 1, \pm 2, \ldots)$. Electrons transporting from terminal 2 into terminals 1 and 3 experience different obstacle barriers. Electrons transporting in the path from terminal 2 to 1 feel the superconducting barrier first, while electrons transporting from terminal 2 to 3 feel the normal obstacle first. The electrons from different paths continue to travel, and at last they meet to form constructive and destructive interference. The unbalanced interference of electrons from different paths results in the Fano peak-valley structure, and the applied gate voltages control the behavior of oscillation structures.

In Fig. 4 we present the transmission coefficient $T_{23}^{Q}(\phi)$ as the function of AB phase $\phi$. This coefficient represents the transport of electrons between terminal 2 and terminal 3. The higher-lower peak structure appears periodically with the period $2 \pi$, and the valleys separating the higher-lower peaks are located at $(2 n+1) \pi,(n=0, \pm 1, \pm 2, \ldots)$. Compared with the Andreev transmission coefficients shown in Figs. 2, and 3, we observe that the normal electron transmission is much larger, and they possess quite different shapes. The oscillation behavior exhibits that the transmission of electrons between the two normal terminals can not be suppressed to zero by changing AB phase. For the completely asymmetric parameters shown by the circled curve, the magnitude of $T_{23}^{Q}(\phi)$ is suppressed, and the peak-valley structure is smeared to form single peak structure. Although this transmission is closely related to the normal electron transport from terminal 2 to 3 , the influence of superconductive terminal is obviously observed by comparing with the solid $(\Delta \neq 0)$ and the dashed $(\Delta=0)$ curves. The modification of superconductive terminal is induced through involving the DOS of the superconductive terminal in the linewidth. The interference of electrons coming from different paths is also efficiently affected by the superconductor.

The transmission coefficient of quasi-particles tunneling from the $\gamma$ th normal terminal to the superconductive terminal is determined by $T_{\gamma 1}(\epsilon)=\Gamma_{\gamma} \Gamma_{1}\left\{\widetilde{N}_{1}(\epsilon)\left[\left|\boldsymbol{G}_{\gamma 1 \sigma, 11}^{\mathrm{r}}(\epsilon)\right|^{2}+\left|\boldsymbol{G}_{\gamma 1 \sigma, 12}^{\mathrm{r}}(\epsilon)\right|^{2}\right]+2 \operatorname{Re}\left[\boldsymbol{G}_{\gamma 1 \sigma, 11}^{\mathrm{r}}(\epsilon) \widetilde{w}_{1}(\epsilon) \boldsymbol{G}_{1 \gamma \sigma, 21}^{\mathrm{a}}(\epsilon)\right]\right\},(\gamma \neq$ 1). The transmission $T_{\gamma 1}(\epsilon)$ requires incident quasi-particles possessing the energy $|\epsilon|>\Delta$. For a normal TQD system, only the first term of it contributes to the transport. As $|\epsilon|>>\Delta$, the effect of superconductive terminal approaches the effect of normal metal terminal. We present the oscillation behavior of the transmission coefficient $T_{21}(\phi)$ as the function of $\phi$ in Fig. 5. The asymmetric Fano resonance structure appears explicitly, and the valleys at $(2 n+1) \pi,(n=0, \pm 1, \pm 2, \ldots)$ are suppressed to zero when the energy levels and gate voltages take the completely asymmetric values (the circled curve). This indicates that the transport is forbidden at these corresponding values of 
$\phi$. The resonant peaks and valleys raise when the symmetric QDs and applied gate voltages are considered. Comparing with Figs. 4 and 5, one observes that the transmission coefficients $T_{23}^{Q}(\phi)$ ( electrons transport between normal terminals) and $T_{21}(\phi)$ (quasi-particle tunnelings) possess the same order. However, the valleys of $T_{23}^{Q}(\phi)$ at $2 n \pi$ are raised compared with the ones in $T_{21}(\phi)$. The asymmetric Fano resonant peaks are generated from unbalanced interference related to the asymmetric paths of electron transport.

\section{A. The case for $e V_{2}=e V_{3}=e V,|e V|<\Delta$}

At zero temperature, the shot noise of the system with equal terminal bias voltages $e V_{2}=e V_{3}=e V$ as $|e V|<\Delta$ is determined by Eq. (8). The shot noise in this regime is contributed by the Andreev reflection, and the correlation of Andreev tunneling with the normal electron transport. We present the variation of shot noise $S_{22}$ versus the AB phase $\phi$ at $e V_{2}=e V_{3}=-0.9$ in Fig. 6. The contentions of normal tunneling and Andreev reflection, as well as the interference of charged particles generate the shot noise with several oscillation structures. For the completely symmetric situation, the oscillation structure exhibits one higher and one lower resonant configuration in a period $2 \pi$ (the solid curve). One resonant peak emerges between the two resonant peaks when we adjust the gate biases and energy levels to form the completely asymmetric situation (the circled curve). This indicates that the oscillation structure is sensitively dependent on the nature of QDs, and external gate voltages. The coherent correlation of the Andreev reflecting and normal tunneling electrons generates the constructive and destructive interference. Although transmission coefficients contain zeros with respect to the AB phase, the shot noise is not blockaded to zero when we adjust the AB phase. This signifies that the shot noise is associated with multiple tunneling effects, and the competition of these transport aspects determines the appearance of shot noise.

The system is controlled by three gate voltages $V_{g i}(i=1,2,3)$, which provide us wider controlling means for adjusting the current characteristics and noise behaviors. We display the shot noise $S_{22}$ with respect to the gate bias $e V_{g 3}$ by setting the other gate biases as definite values exhibited in Fig. 7 . When the gate bias $e V_{g 3}$ approaches zero, an inverse resonance appears to show the minimum value of shot noise $0.01 S_{0}$. The concrete appearance of shot noise is modified with respect to supplying different values of energy levels and the other gate voltages. For the system with equal energy levels and equal gate biases ( solid curve), the shot noise reaches its maximum value $0.36 S_{0}$ at about $e V_{g 3}=-6$. The shot noise behaves asymmetrically with lower right apex value $0.3 S_{0}$ at about $e V_{g 3}=5$. The asymmetric configuration is strengthened as the energy levels and gate voltages are deviated from the equally chosen ones.

The Fano factor versus gate bias $e V_{g 3}$ for the system with equal terminal biases $e V_{2}$ and $e V_{3}$ in the Andreev reflection 
regime is presented in Fig. 8. Varying the gate voltages in the case as $e V_{2}=e V_{3}=e V$, and $|e V|<\Delta$, the Fano factor remains $F_{2}<1$, and the type of sub-Poissonian noise exhibits the suppression effect. An inverse resonant dip appears as $e V_{g 3}$ approaches -1, and the asymmetric Fano resonant peak-valley structure is displayed obviously for equally chosen parameters ( the solid curve). The asymmetric structure can be enhanced as we adjust the energy levels of QDs and gate bias $e V_{g i}$. As $E_{1}=0.1, E_{2}=-0.1, E_{3}=0.2$, and $e V_{g 1}=e V_{g 2}=0.1$, the Fano factor reaches its maximum value $F_{2} \approx 0.75$ at about $e V_{g 3}=-5$ (the dotted curve). The Factor reaches its minimum value $F_{2} \approx 0.23$ at about $\mathrm{eV}_{g 3}=5$ with the changing rate $69 \%$ for the dotted curve. The left apex and right peak-valley structure is modified drastically to form double-peak structure by changing the parameters to $e V_{g 1}=0.1, e V_{g 2}=-0.2($ the circled curve), while keeping the energy levels unchanged as the ones of dotted curve.

\section{B. The case for $\left|e V_{2}\right|<\Delta,\left|e V_{3}\right|>\Delta$}

For the system applied with different terminal bias voltages $V_{i}$, the electron transmissions between different terminals induce additional current correlations. These current correlations enhance the shot noise described by Eq. (7). We present the shot noise $S_{22}$ of terminal 2 by changing the gate bias $e V_{g 3}$ in Fig. 9 when $e V_{2}=-0.9, e V_{3}=1.2$. The resonant structure of shot noise appears with the magnitude $S_{22} \approx 0.48 S_{0}$ as $e V_{g 3} \approx 0$ for the hybrid TQD interferometer. The dependence of shot noise on different energy levels and gate voltages is depicted by different curves. The competition of enhancement and suppression of shot noise is attributed to: (a) the self-correlations of Andreev reflection, normal electrons, and quasi-particles; (b) the cross-correlation of Andreev reflection and normal electrons; (c) the cross-correlation of normal electrons and quasi-particles; (d) the cross-correlation of Andreev reflection and quasi-particles. The structure of shot noise exhibits Fano asymmetric resonance deviating from the Breit-Wigner form obviously. The shot noise of normal system (the dashed curve) is given by $\Delta \rightarrow 0$, which is smaller than that of the hybrid system. This signifies that the cross-correlations among Andreev reflection, normal and quasi-particles enhance the shot noise of the TQD interferometer.

The Fano factor $F_{2}$ in the regime $e V_{2}=-0.9, e V_{3}=1.2$ is exhibited in Fig. 10 as the function of $e V_{g 3}$. The resonant behavior is displayed with the magnitude about $F_{2} \approx 1.62$ for the hybrid system shown by different curves. The affection of energy levels and gate voltages on the Fano factor exhibits indistinctly around the resonant regime. However, it varies drastically in the regime away from the resonant peak. The enhancement of shot noise comes from the cross-correlations of currents in different normal terminals, and the Andreev reflecting currents induced by the superconductive terminal. The shot noise reaches super-Poissonian form with $F_{2}>1$ when the absolute value of gate voltage $\left|e V_{g 3}\right|<5$. The Fano factor of normal metal system is presented by the dashed curve. Although in large 
regime of $e V_{g 3}$ the shot noise belongs to sub-Poissonian, the magnitude of Fano factor can still reach $F_{2} \approx 1.15$ around the resonant regime. In this regime, the shot noise for the normal metal system belongs to super-Poissonian. This indicates that the influence of superconductor on noise results in larger Fano factor. The coherent current exhibits simple relation in this regime. The complicated correlations of different current components generate the enhancement of shot noise.

\section{Concluding Remarks}

The shot noise formula of a hybrid TQD interferometer has been derived by employing the NGF method. The shot noise is contributed by the self-correlation of Andreev reflecting current in the $\gamma$ th terminal, the cross-correlation of Andreev reflection between $\gamma$ and $\gamma^{\prime}$ normal terminals, and electron transport between different normal terminals. The behaviors of shot noise and Fano factor are intimately dependent on the terminal biases, where sub-Poissonian and super-Poissonian noise appears in different regimes of $e V_{\gamma}$. In the regime $e V_{\gamma}=e V_{\gamma^{\prime}}=e V\left(\gamma \neq \gamma^{\prime}\right)$, and $|e V|<\Delta$, the shot noise presents sub-Poissonian contributed by the Andreev reflection, and correlation of Andreev tunneling with the normal electron transport. The inverse resonance structure displays when we adjust one of the gate voltages by setting the other gate voltages. The asymmetric structure can be enhanced as we adjust the energy levels of QDs and the gate bias $e V_{g i}$. Although the transmission coefficients can be blockaded to zero when we change the AB phase, the corresponding shot noise can not be suppressed to zero. Super-Poissonian shot noise exhibits in the regime $\left|e V_{\gamma}\right|<\Delta,\left|e V_{\gamma^{\prime}}\right|>\Delta,\left(\gamma \neq \gamma^{\prime}\right)$, where the resonance structure appears with respect to one of the gate voltages. The competition of self-correlation and cross-correlation between different current components achieves the enhancement resonant shot noise.

\section{Acknowledgments}

We gratefully acknowledge the support by Research Grant Council from the SAR Government of Hong Kong under the Grant No. HKU 705611P, and the National Natural Science Foundation of China under the Grant No. 11175015.

1 G. C. Aers, et al, Phys. Rev. B 86(2012)045316.

${ }^{2}$ C. Bruder, R. Fazio, and H. Schoeller, Phys. Rev. Lett. 76(1996) 114; K. Kang, Phys. Rev. B 59(1999) 4608.

${ }^{3}$ W. Hofstetter, J. König, and H. Schoeller, Phys. Rev. Lett. 87 (2001) 156803.

${ }^{4}$ H. Gao, and H. K. Zhao, EPL 83 (2008) 38002; H. Gao, and H. K. Zhao, Phys. Lett. A $377(2013) 1210$. 
${ }^{5}$ B. Kubala, and J. König, Phys. Rev. B 65 (2002) 245301.

${ }^{6}$ I. Weymann, Phys. Rev. B 78 (2008) 045310; P. Trocha, I. Weymann, and J. Barnaś, Phys. Rev. B 80(2009) 165333.

7 B. Hiltscher, M. Governale, and J. König, Phys. Rev. B 81 (2010) 085302.

${ }^{8}$ S. Sasaki, H. Tamura, T. Akazaki, and T. Fujisawa, Phys. Rev. Lett. 103(2009) 266806; R. Zitko, Phys. Rev. B 81 (2010) 115316.

${ }^{9}$ H. K. Zhao, and L. L. Zhao, Eur. Phys. J. B 79(2011) 485 ; L. L. Zhao, H. K. Zhao, and J. Wang, Phys. Lett. A 376(2012) 1849.

${ }^{10}$ F. Delgado, et al, Phys. Rev. Lett. 101(2008) 226810.

11 S. Amaha, et al, Phys. Rev. B 85(2012) 081301(R).

12 B. Michaelis, C. Emary, and C. W. J. Beenakker, Europhys. Lett. 73(2006) 677; C. Emary, Phys. Rev. B 76(2007) 245319.

13 F. Domínguez, G. Platero, and S. Kohler, Chem. Phys. 375(2010) 284.

14 Ya. M. Blanter, M. Büttiker, Phys. Rep. 336(2000) 1.

15 M. H. Pedersen, and M. Büttiker, Phys. Rev. B 58 (1998) 12993.

16 J. Lehmann, S. Kohler, and P. Hänggi, J. Chem. Phys. 118(2003) 3283; S. Kohler, J. Lehmann, P. Hänggi, Phys. Rep. 406(2005) 379 .

17 F. Lefloch, C. Hoffmann, M. Sanquer, and D. Quirion, Phys. Rev. Lett., 90 (2003) 067002.

18 Y. C. Chung, M. Heiblum, and V. Umansky, Phys. Rev. Lett. 91(2003) 216804.

19 M. Dolev, M. Heiblum, V. Umansky, A. Stern, and D. Mahalu, Nature (London)452 (2008) 829.

20 M. Dolev, et al, Phys. Rev. B 81(2010) 161303(R).

21 S. Kafanov, and P. Delsing, Phys. Rev. B 80(2009) 155320.

${ }^{22}$ E. Onac, et al , Phys. Rev. Lett. 96(2006) 176601.

${ }^{23}$ E. Onac, F. Balestro, B. Trauzettel, C. F. J. Lodewijk, and L. P. Kouwenhoven, Phys. Rev. Lett. 96(2006) 026803.

${ }^{24}$ S. Gustavsson, et al, Phys. Rev. Lett. 99(2007) 206804.

25 G. E. Blonder, M. Tinkham, and T. M. Klapwijk, Phys. Rev. B 25(1982) 4515.

${ }^{26}$ C. W. J. Beenakker, Phys. Rev. Lett. 97, (2006) 067007; C. W. J. Beenakker, Rev. Mod. Phys. 80(2008) 1337.

27 Q. Zhang, D. Fu, B.G. Wang, R. Zhang, and D. Y. Xing, Phys. Rev. Lett. 101 (2008) 047005.

28 S.G. Cheng, H. Zhang, and Q. F. Sun, Phys. Rev. B 83 (2011) 235403.

29 S. Russo, M. Kroug, T. M. Klapwijk, and A. F. Morpurgo, Phys. Rev. Lett. 95(2005) 027002.

${ }^{30}$ P. Cadden-Zimansky, and V. Chandrasekhar, Phys. Rev. Lett. 97(2006) 237003.

31 D. S. Golubev, and A. D. Zaikin, Phys. Rev. B 76 (2007) 184510.

32 A. Levy Yeyati, F. S. Bergeret, A. Martín-Rodero, and T. M. Klapwijk, Nat. Phys. 3(2007) 455.

33 J. Wei, and V. Chandrasekhar, Nat. Phys. 6(2010) 494-498. 
${ }^{34}$ Q. F. Sun, J. Wang, and T. H. Lin, Phys. Rev. B 59(1999) 3831; 59(1999) 13126.

${ }^{35}$ H.K. Zhao, and J. Wang, Phys. Rev. B 64(2001) 094505; H.K. Zhao, G.V. Gehlen, Phys. Rev. B 58(1998) 13660.

36 J. Peng, B. G. Wang, and D. Y. Xing, Phys. Rev. B 71, (2005) 214523.

37 R. López, M. S. Choi, R. Aguado, Phys. Rev. B 75(2007) 045132; R. Zitko, M. Lee, R. López, R. Aguado, M. S. Choi, Phys. Rev. Lett. 105 (2010) 116803.

38 L. Bai, Y. J. Wu, and B. G. Wang, Phys. Status Solidi B 247(2010) 335.

39 M. P. Anantram, S. Datta, Phys. Rev. B 53(1996) 16390.

40 L. Wan, Y. Wei, and J. Wang, Nanotechology, 17(2006)489.

${ }^{41}$ H. K. Zhao, Phys. Lett. A, 299(2002) 262; H. K. Zhao, J. Wang, and Q. Wang, EPL, 99(2012) 48005.

42 A. P. Jauho, N. S. Wingreen, and Y. Meir, Phys. Rev. B 50(1994) 5528.

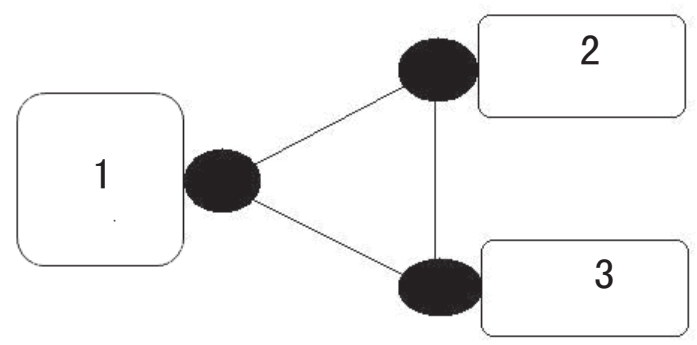

FIG. 1: The schematic structure of the TQD system. The superconductor is connected to QD 1, while the normal terminals are connected to QD 2, and QD 3. 


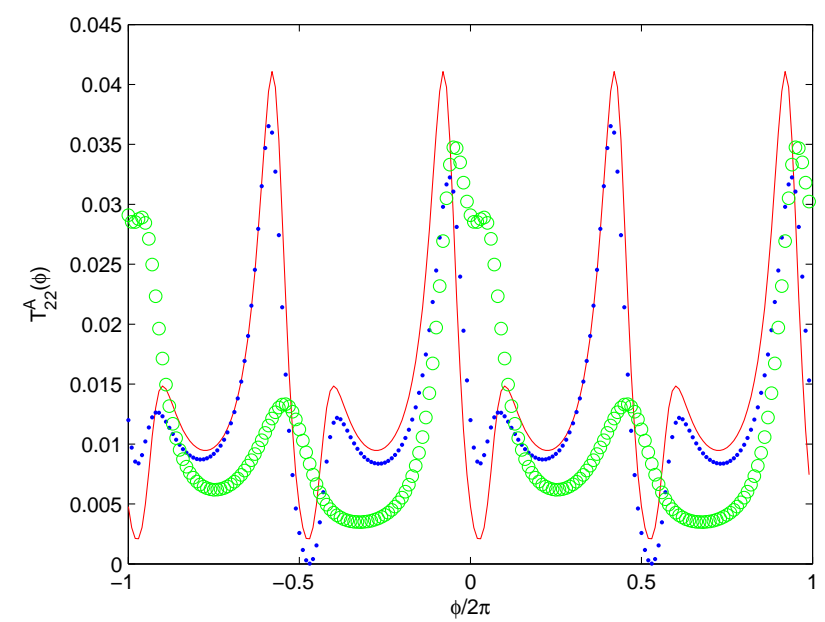

FIG. 2: (Color online) The Andreev transmission coefficient $T_{22}^{A}(\phi)$ versus $\phi$ at $\epsilon=0.9$. The parameters are chosen as $E_{1}=$ $E_{2}=E_{3}=0.1$, and $e V_{g 1}=e V_{g 2}=e V_{g 3}=0.1$ for the solid curve; $E_{1}=0.1, E_{2}=-0.1, E_{3}=0.2$, and $e V_{g 1}=e V_{g 2}=e V_{g 3}=0.1$ for the dotted curve; $E_{1}=0.1, E_{2}=-0.1, E_{3}=0.2$, and $e V_{g 1}=0.3, e V_{g 2}=0.1, e V_{g 3}=-0.3$ for the circled curve.

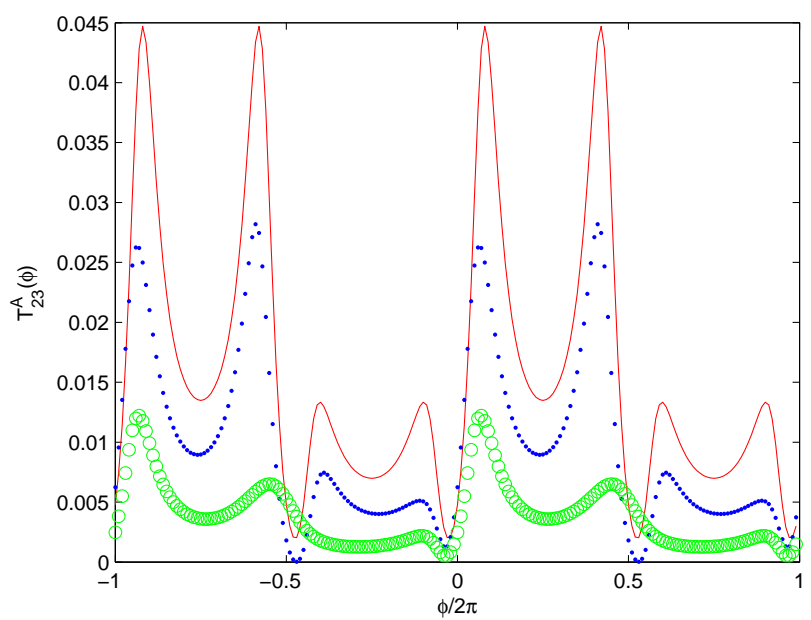

FIG. 3: (Color online) The Andreev transmission coefficient $T_{23}^{A}(\phi)$ versus $\phi$ at $\epsilon=0.9$. The parameters are chosen as $E_{1}=$ $E_{2}=E_{3}=0.1$, and $e V_{g 1}=e V_{g 2}=e V_{g 3}=0.1$ for the solid curve; $E_{1}=0.1, E_{2}=-0.1, E_{3}=0.2$, and e $e V_{g 1}=e V_{g 2}=e V_{g 3}=0.1$ for the dotted curve; $E_{1}=0.1, E_{2}=-0.1, E_{3}=0.2$, and $e V_{g 1}=0.3, e V_{g 2}=0.1, e V_{g 3}=-0.3$ for the circled curve. 


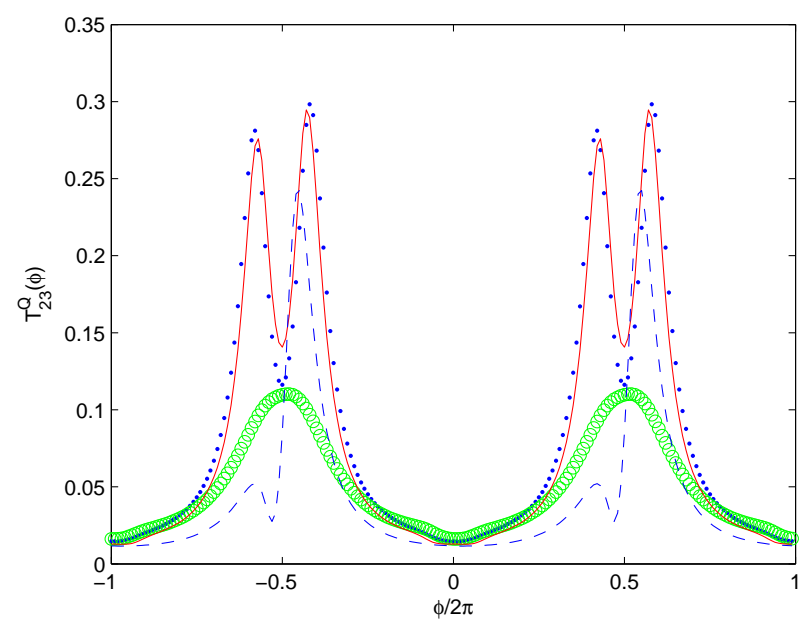

FIG. 4: (Color online) The transmission coefficient $T_{23}^{Q}(\phi)$ versus $\phi$ at $\epsilon=0.9$. The parameters are chosen as $E_{1}=E_{2}=E_{3}=$ 0.1 , and $e V_{g 1}=e V_{g 2}=e V_{g 3}=0.1$ for the solid curve; $E_{1}=0.1, E_{2}=-0.1, E_{3}=0.2$, and $e V_{g 1}=e V_{g 2}=e V_{g 3}=0.1$ for the dotted curve; $E_{1}=0.1, E_{2}=-0.1, E_{3}=0.2$, and $e V_{g 1}=0.3, e V_{g 2}=0.1, e V_{g 3}=-0.3$ for the circled curve. The dashed curve is depicted for the normal system by setting $\Delta=0$, and the parameters are chosen as the ones of the solid curve.

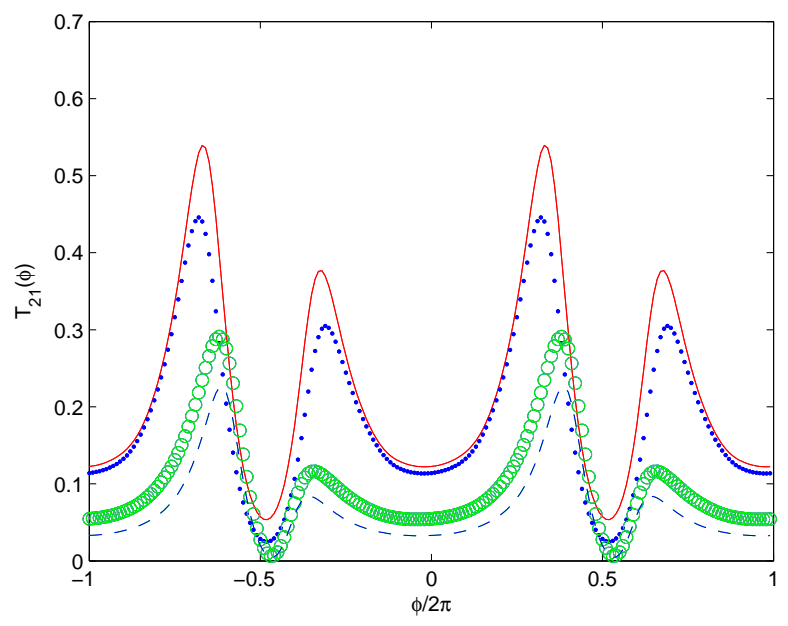

FIG. 5: (Color online) The transmission coefficient $T_{21}(\phi)$ versus $\phi$ at $\epsilon=1.2$. The parameters are chosen as $E_{1}=E_{2}=E_{3}=$ 0.1 , and $e V_{g 1}=e V_{g 2}=e V_{g 3}=0.1$ for the solid curve; $E_{1}=0.1, E_{2}=-0.1, E_{3}=0.2$, and $e V_{g 1}=e V_{g 2}=e V_{g 3}=0.1$ for the dotted curve; $E_{1}=0.1, E_{2}=-0.1, E_{3}=0.2$, and $e V_{g 1}=0.3, e V_{g 2}=0.1, e V_{g 3}=-0.3$ for the circled curve. The dashed curve is depicted for the normal system by setting $\Delta=0$, and the parameters are chosen as the ones of the circled curve. 


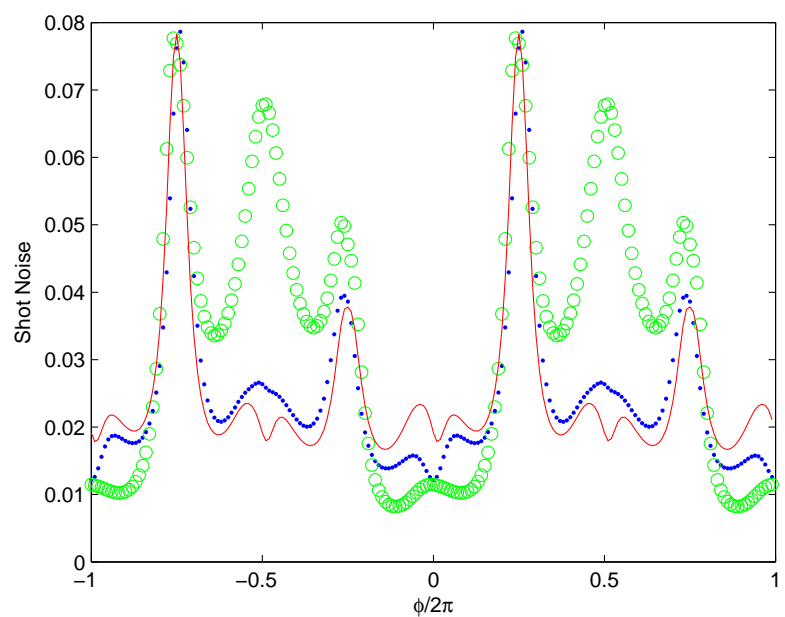

FIG. 6: (Color online) The shot noise $S_{22}$ versus $\phi$ at $e V_{2}=e V_{3}=-0.9$. The parameters are chosen as $E_{1}=E_{2}=E_{3}=0.1$, and $e V_{g 1}=e V_{g 2}=e V_{g 3}=0.1$ for the solid curve; $E_{1}=0.1, E_{2}=-0.1, E_{3}=0.2$, and $e V_{g 1}=e V_{g 2}=e V_{g 3}=0.1$ for the dotted curve; $E_{1}=0.1, E_{2}=-0.1, E_{3}=0.2$, and $e V_{g 1}=0.3, e V_{g 2}=0.1, e V_{g 3}=-0.3$ for the circled curve.

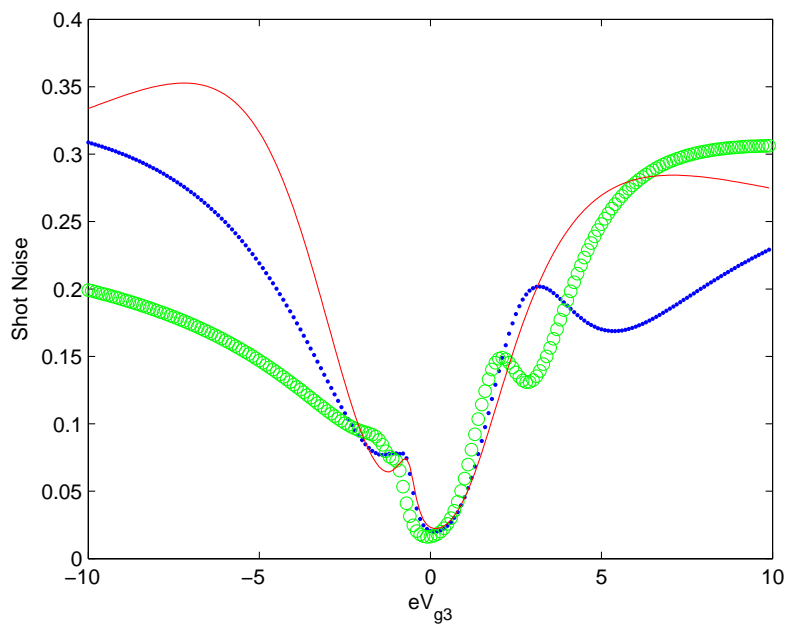

FIG. 7: (Color online) The shot noise $S_{22}$ versus $e V_{g 3}$ at $e V_{2}=e V_{3}=-0.9$, and $\phi=\pi / 3$. The parameters are chosen as $E_{1}=E_{2}=E_{3}=0.1, e V_{g 1}=e V_{g 2}=0.1$ for the solid curve; $E_{1}=0.1, E_{2}=-0.1, E_{3}=0.2$, and $e V_{g 1}=e V_{g 2}=0.1$ for the dotted curve; $E_{1}=0.1, E_{2}=-0.1, E_{3}=0.2$ with $e V_{g 1}=0.3, e V_{g 2}=0.1$ for the circled curve. 


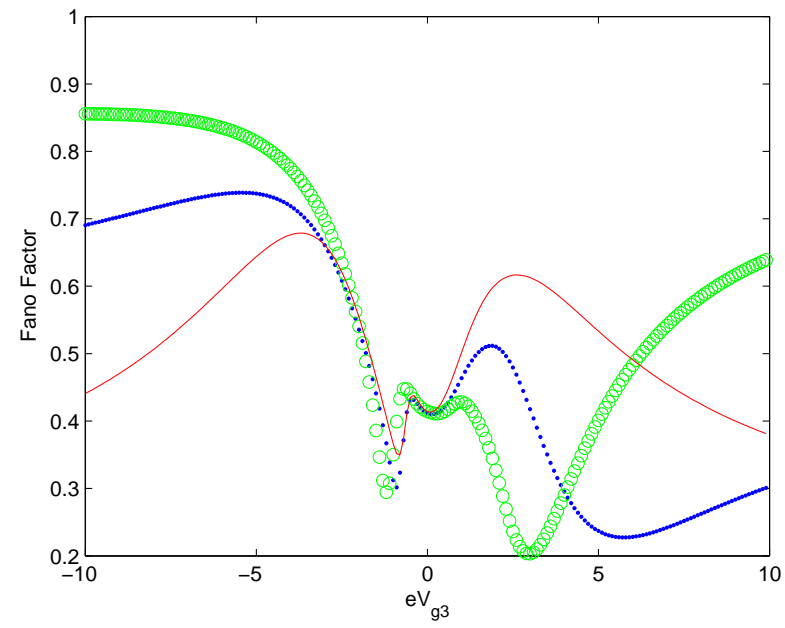

FIG. 8: (Color online) The Fano factor $F_{2}$ versus $e V_{g 3}$ at $e V_{2}=e V_{3}=-0.9$, and $\phi=\pi / 3$. The parameters are chosen as $E_{1}=E_{2}=E_{3}=0.1, e V_{g 1}=e V_{g 2}=0.1$ for the solid curve; $E_{1}=0.1, E_{2}=-0.1, E_{3}=0.2$, and $e V_{g 1}=e V_{g 2}=0.1$ for the dotted curve; $E_{1}=0.1, E_{2}=-0.1, E_{3}=0.2$ with $e V_{g 1}=0.3, e V_{g 2}=0.1$ for the circled curve.

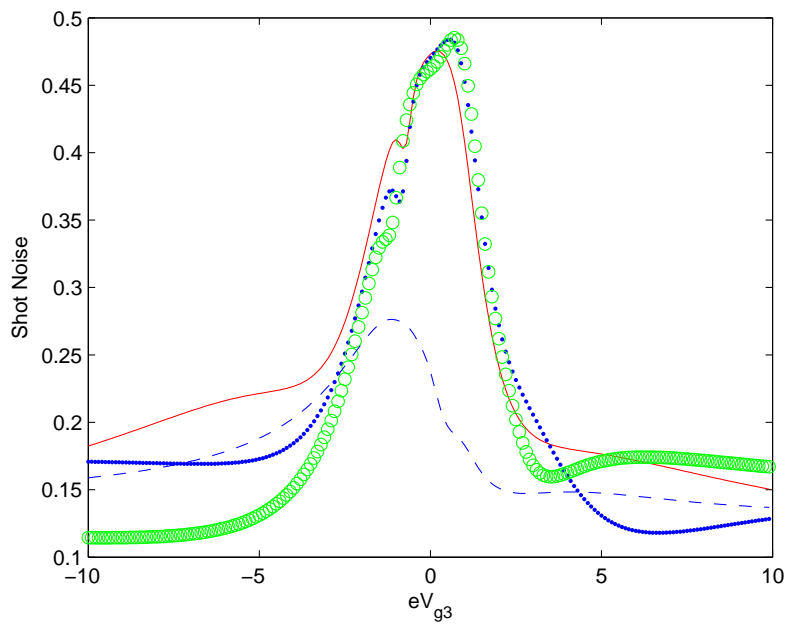

FIG. 9: (Color online) The shot noise $S_{22}$ versus $e V_{g 3}$ at $e V_{2}=-0.9, e V_{3}=1.2$, and $\phi=\pi / 3$. The parameters are chosen as $E_{1}=E_{2}=E_{3}=0.1, e V_{g 1}=e V_{g 2}=0.1$ for the solid curve; $E_{1}=0.1, E_{2}=-0.1, E_{3}=0.2$, and $e V_{g 1}=e V_{g 2}=0.1$ for the dotted curve; $E_{1}=0.1, E_{2}=-0.1, E_{3}=0.2$ with $e V_{g 1}=0.3, e V_{g 2}=0.1$ for the circled curve. The dashed curve is related to $\Delta=0$ with parameters chosen as those in solid curve. 


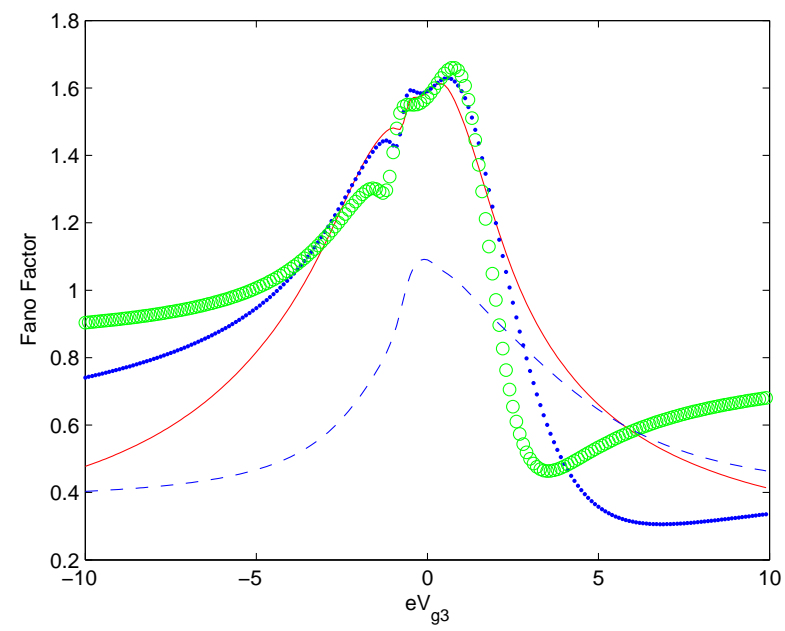

FIG. 10: (Color online) The Fano factor $F_{2}$ versus $e V_{g 3}$ at $e V_{2}=-0.9, e V_{3}=1.2$, and $\phi=\pi / 3$. The parameters are chosen as $E_{1}=E_{2}=E_{3}=0.1, e V_{g 1}=e V_{g 2}=0.1$ for the solid curve; $E_{1}=0.1, E_{2}=-0.1, E_{3}=0.2$, and $e V_{g 1}=e V_{g 2}=0.1$ for the dotted curve; $E_{1}=0.1, E_{2}=-0.1, E_{3}=0.2$ with $e V_{g 1}=0.3, e V_{g 2}=0.1$ for the circled curve. The dashed curve is related to $\Delta=0$ with parameters chosen as those in solid curve. 